\title{
Chemical abundances in planetary nebulae in three different galaxies
}

\author{
Miriam Peña \\ Instituto de Astronomía, Universidad Nacional Autónoma de México
}

\begin{abstract}
We analyze the PNe chemical behavior in three different galaxies, two dwarf irregulars and one spiral. Different behaviors are found. In the very low metallicity galaxy NGC 3109, PNe analyzed appear 0.39 dex O-richer than HII regions, while $\mathrm{Ar} / \mathrm{H}$ ratio is, in average, 0.15 dex poorer. We interpret this as an evidence of significant $\mathrm{O}$ dredge-up in these LIMS, born in a very low metallicity environment. In $\mathrm{NGC} 6822$, with a present metallicity $12+\log \mathrm{O} / \mathrm{H}=8.06$, two PN populations were found. A young one, with abundances similar to those in HII regions and an old population, with metallicities a factor of two lower. In this case no strong evidence for $\mathrm{O}$ dredge-up in LIMS is found. Therefore, metallicities lower than $12+\log \mathrm{O} / \mathrm{H}=7.7$ are required for an efficient $\mathrm{O}$ dredge-up. From our preliminary analysis of the abundances of $\mathrm{PNe}$ in NGC 300 we find that they are similar to the abundances in HII regions. Apparently, the PNe analyzed belong to a young population. Very similar abundance gradients, with galactocentric distance, are found for HII regions and for PNe.
\end{abstract}

Keywords. planetary nebulae: general, galaxies: individual (NGC 3109, NGC 6822, NGC 300), ISM: abundances, etc.

\section{Introduction}

By using the on-line off-line technique in the [O III] 5007 and $\mathrm{H} \alpha$ lines, we have searched for emission line nebulae in three different galaxies: NGC 3109, a dwarf irregular at the Local Group edge; NGC 6822, a dwarf irregular of the Local Group and NGC 300, a Scd-type nearby spiral galaxy. This technique allows us to detect HII regions, planetary nebulae (PNe) and other emission line objects. To select PN candidates we employed the criteria described in Peña et al. (2007a) and Hernández-Martínez et al. (2009a) which have been very successful to distinguish PNe from compact HII regions. So far we have detected 20 PNe in NGC 3109 (Peña et al. 2007a), 26 PNe in NGC 6822 (HernándezMartínez et al., 2009a) and more than 60 PN candidates in NGC 300 (Peña et al., in preparation).

Follow-up spectroscopy of a sub-sample of emission line objects has been performed, to verify their nature and to determine their chemical abundances. Data have been collected with the VLT-FORS and Gemini GMOS multi-object spectrographs. Exposure times were long enough to detect with signal-to-noise better than 3 , the diagnostic-lines [O III] 4363 for electron temperature and [SII] 6719,6731, for electron density. This is extremely important in order to calculate reliable ionic abundances, based on electron temperature and density measurements.

Abundances of $\mathrm{He}, \mathrm{O}, \mathrm{N}, \mathrm{Ne}, \mathrm{Ar}$ and $\mathrm{S}$ were calculated for most of our objects from the ionic abundances determined and using the ionization correction factors suggested by Kingsburgh \& Barlow (1994). Results for NGC 3109 were presented in Peña et al. (2007b), and for NGC 6822 in Hernández-Martínez et al. (2009b). A summary of these results are presented in Table 1, where PN and HII region abundances are presented separately. The 
Table 1. Total abundances for PNe and HII regions in NGC 3109, NGC 6822 and the Magellanic Clouds

\begin{tabular}{llllll}
\hline object & $12+\log \mathrm{O} / \mathrm{H}$ & $\mathrm{N} / \mathrm{O}$ & $\mathrm{Ne} / \mathrm{O}$ & $\mathrm{Ar} / \mathrm{H}$ & references $^{1}$ \\
\hline NGC3109-HII & $7.75 \pm 0.10$ & $-1.28 \pm 0.05$ & $-0.88 \pm 0.06$ & $2.01 \pm 0.15$ & PSR \\
NGC3109-PNe & $8.14 \pm 0.20$ & $-0.80 \pm 0.20$ & $-0.80 \pm 0.15$ & $-2.45 \pm 0.25$ & PSR \\
NGC6822-HII & $8.06 \pm 0.04$ & $-1.34 \pm 0.15$ & $-0.73 \pm 0.07$ & $-2.18 \pm 0.10$ & H-M et al. \\
NGC6822-young PNe & $8.08 \pm 0.12$ & $-0.50 \pm 0.50$ & $-0.78 \pm 0.08$ & $-2.26 \pm 0.20$ & H-M et al. \\
NGC6822-old PNe & $7.70 \pm 0.20$ & $-0.88 \pm 0.30$ & $-0.77 \pm 0.12$ & $-2.26 \pm 0.20$ & H-M et al. \\
LMC-HII & 8.40 & -1.50 & -0.80 & -2.20 & D89, G99 \\
LMC-PNe & 8.33 & -0.88 & -0.79 & -2.40 & LD06 \\
SMC-HII & 8.00 & -1.50 & -0.80 & -2.10 & D89, G99 \\
SMC-PNe $^{2}$ & 8.09 & -0.98 & -0.93 & -2.58 & LD06 \\
\hline
\end{tabular}

${ }^{1}$ Ref: PSR: Peña et al. (2007); H-M et al: Hernandez-Martinez et al. 2009b;

D89: Dennefeld (1989); G99: Garnett (1999); LD06: Leisy \& Dennefeld (2006)

${ }^{2}$ Type I PNe not included

analysis for NGC 300 objects is in preparation (Stasińska et al., in preparation). In the following we discuss these results comparatively.

\section{The chemical abundances of $\mathrm{HII}$ regions and $\mathrm{PNe}$}

\subsection{The case for NGC 3109}

This irregular late-type spiral galaxy, is the dominant one in the Antlia-Sextans group. It is known to be extremely metal poor. $12+\log \mathrm{O} / \mathrm{H}$ abundances of about 7.74 were reported by Lee et al. (2003) for a few HII regions. In our work we analyzed 12 HII regions distributed over the whole extension of the galaxy. The chemical composition derived for these objects shows a very homogeneous galaxy, with an average $12+\log \mathrm{O} / \mathrm{H}$ $=7.77 \pm 0.07$ (a factor of about 2 poorer than the SMC, see Table 1), in very good agreement with the value $7.76 \pm 0.07$ derived by Evans et al. (2007) for B super-giants. Other well determined elements like $\mathrm{N}$ and $\mathrm{Ne}$ also show very little scatter confirming the homogeneity of the present interstellar medium in this galaxy.

On the other hand, the sample of 8 analyzed PNe shows a very different abundance pattern. This PN sample seems entirely composed of O-enriched objects, being their oxygen abundances, on average, 0.39 dex larger than those in HII regions. On the other hand, their $\mathrm{Ne} / \mathrm{O}$ ratios have the same average value as the HII regions. The O-enrichment found in $\mathrm{PNe}$, as compared to HII regions, is favoring evolutionary models that predict $\mathrm{O}$ dredge-up in low metallicity PN progenitors (e.g., Marigo 2001), and the similar average in $\mathrm{Ne} / \mathrm{O}$ ratios would indicate that also $\mathrm{Ne}$ is affected by the evolution in these stars. This is the first occasion where such an evidence in favor of $\mathrm{O}$ enrichment, during third dredge-up events, is observed in a significant sample of PNe. Other examples are: the only PN known in Sextans A (Kniazev et al. 2005; Magrini et al. 2005) and the few most metal-poor PNe in the SMC (Leisy \& Dennefeld 2006). The only two PNe for which N was determined show no extreme N enrichment. Regarding Ar and S, these abundance determinations are very uncertain, but they appear under-abundant in PNe as compared to HII regions, thus confirming that $\mathrm{O}$ and Ne are enriched. This should be studied with better precision. It would be fruitful to derive reliable Ar and $\mathrm{S}$ abundances.

\subsection{The abundance behavior in NGC 6822}

This galaxy is one of the closest gas-rich dwarfs in our vicinity. Located half-way between our galaxy and Andromeda, it seems well suited for chemical evolution studies as apparently it is isolated. However it is a complicated system. Its stellar content spreads 


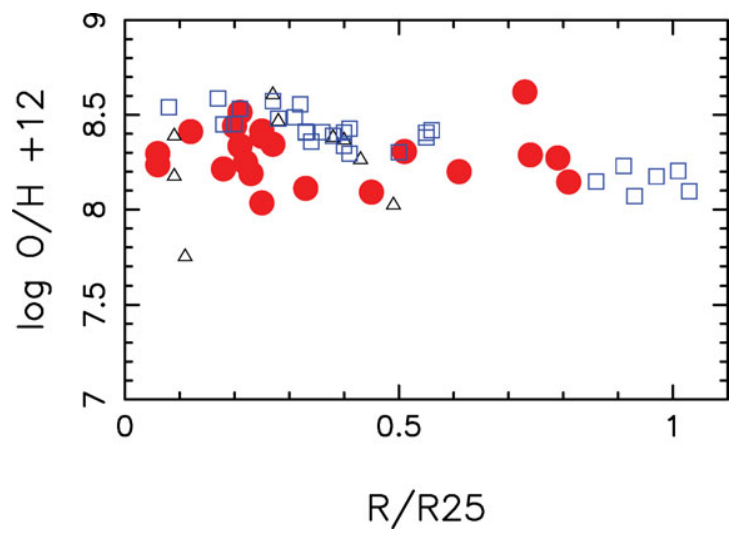

Figure 1. Oxygen abundance as a function of the normalized galactocentric distance for PNe (dots) and HII regions (open squares, data from Bresolin et al. 2009) in NGC 300. A similar chemical gradient is evident.

in a zone of about $3 \times 3 \mathrm{kpc}$ and it has a huge rotating $\mathrm{HI}$ disk with dimensions of $6 \times 14$ kpc. In addition a spheroidal distribution of $\mathrm{C}$ stars has also been found.

We analyzed sample of eleven $\mathrm{PNe}$ and three compact $\mathrm{HII}$ regions. $\mathrm{He}, \mathrm{O}, \mathrm{N}, \mathrm{Ne}$, Ar and $\mathrm{S}$ abundances were derived for most of the nebulae. The results are reported in Hernández-Martínez et al. (2009b). Combining our results for the compact HII regions with others from the literature, we have found that the present interstellar medium in this galaxy is homogeneous at least in its three central parsecs, showing a value $12+\log$ $\mathrm{O} / \mathrm{H}=8.06 \pm 0.04$. Other elements like $\mathrm{N}, \mathrm{Ne}$ and Ar show also a homogeneous galaxy. The situation is different for the PN sample where we detected two kind of objects. Six PNe of the analyzed sample present abundance pattern similar to HII regions, with an average of $12+\log \mathrm{O} / \mathrm{H}=8.08 \pm 0.12$. This sample includes two Peimbert Type I PN, extremely N-rich. We have identified these PNe as young objects. The other 5 PNe show abundances a factor of 2 poorer than the young group, with a $12+\log \mathrm{O} / \mathrm{H}$ average of $7.70 \pm 0.20$. An age of about 6 Gyr can be attributed to these objects. Although metal poor, these $\mathrm{PNe}$ do not show evidence of O-enrichment as their $\mathrm{Ar} / \mathrm{O}$ abundance ratios are similar to those in the young PNe and HII regions. Thus O dredge-up has not occurred in their central stars. Therefore, apparently a metallicity lower than $12+\log \mathrm{O} / \mathrm{H}=7.7$ is needed for significant $\mathrm{O}$ dredge-up in low-intermediate mass stars.

Chemical evolution models, to reproduce the chemical behavior of PNe and HII regions in this galaxy, have been computed. They will be presented elsewhere (HernándezMartínez et al., in preparation).

\subsection{NGC 300, a Sc-type nearby spiral}

The search for PN candidates and follow-up spectroscopy in this galaxy, were performed with VLT-FORS 2 on August, 2006 (program ID 077.B-0430B). This galaxy extends over an angle of about 25 arcmin in the sky and, as said in the introduction, two zones were analyzed, a central field and an outskirts one, each one of $6.8 \times 6.8$ squared arcmin. In total we have detected $60 \mathrm{PN}$ candidates (about 40 in the central region and 20 in the outskirts) and follow-up spectroscopic data were obtained for 22 of them. A few compact HII regions were also analyzed. The 22 candidates were confirmed as PNe, which implies that our method for choosing candidates is very reliable.

Preliminary chemical abundances have been derived for the PNe for which [OIII] electron temperatures were measured. Our results for $\mathrm{O}$ abundances are shown in Fig. 1 
(kindly provided by Grazyna Stasińska), where $12+\log \mathrm{O} / \mathrm{H}$ is plotted as a function of the galactocentric distance normalized to $\mathrm{R}_{25}$. In this figure we include the abundances determined for a large sample of HII regions by Bresolin et al. (2009). Some of our compact HII regions are also shown.

From this figure it is clear that PNe analyzed in NGC 300 show $\mathrm{O}$ abundances similar to HII regions, all over the galaxy. In this sense, all our PNe behave as young objects. No old under-abundant PN population has been detected. This is most probably a selection effect, because, to perform a reliable spectroscopical study, only the brightest (and probably the youngest) PN candidates were observed. NGC 300, at a distance of $1.88 \mathrm{kpc}$ (Gieren et al. 2005) is much farther away than NGC 6822. The abundance gradient with galactocentric distance, observed for HII regions, is also present in our PN sample, with very similar value. This gradient has been computed to be $-0.077 \pm 0.06$ by Bresolin et al. (2009)

\section{Conclusions}

We have analyzed the PNe chemical behavior in three different galaxies, two dwarf irregulars and one spiral. Different behaviors are found. So far, a few conclusions can be drawn:

In the very low metallicity galaxy NGC 3109 (present metallicity $12+\log \mathrm{O} / \mathrm{H}=7.75 \pm$ 0.10), PNe analyzed appear 0.39 dex O-richer than HII regions, while $\mathrm{Ar} / \mathrm{H}$ ratio is, in average, 0.15 dex poorer. We interpret this as an evidence of significant $\mathrm{O}$ dredge-up in these low-intermediate mass stars (LIMS), that were born in a very low metallicity environment some Gyr ago.

In NGC 6822, with a present metallicity $12+\log \mathrm{O} / \mathrm{H}=8.06$, two $\mathrm{PN}$ populations were found. Young PNe have similar abundances than HII regions. A population of old $\mathrm{PNe}$ shows metallicity a factor of two lower. In this case no strong evidence for O dredge-up in LIMS is found. Therefore, metallicities lower than $12+\log \mathrm{O} / \mathrm{H}=7.7$ are required for an efficient $\mathrm{O}$ dredge-up.

Both irregular galaxies are chemically homogeneous.

From our preliminary analysis of the chemical composition in PNe in NGC 300 we find that they show similar abundances than HII regions. Apparently, the PNe analyzed belong to a young population. Clear abundance gradients with galactocentric distance, are found for HII regions and for PNe. Both gradients are very similar.

This work received financial support from DGAPA-UNAM (ID 112708).

\section{References}

Bresolin, F Gieren, W., \& Kudritzki, R.-P. 2009, ApJ, 700, 309

Evans, C. J., Bresolin, F., Urbaneja, M. A., et al. 2007, ApJ, 659, 1198

Gieren, W., Pietrzyński, G., Soszyński, I., Bresolin, F., et al. 2005, ApJ, 628, 695

Hernández-Martínez, L. \& Peña, M. 2009a, A\&A, 495, 447

Hernández-Martínez, L., Peña, M., Carigi,L., \& García-Rojas, J. 2009b, A\&A, 505, in press

Kingsburgh, R. \& Barlow, M. 1994, MNRAS, 271, 257

Lee H., McCall M. L., Kingsburgh R. L., Ross R., \& Stevenson C. C. 2003, AJ, 125, 146

Kniazev, A. Y., Grebel, E. K., Pustilnik, S. A., Pramskij, A. G., \& Zucker, D. B. 2005, AJ, 130, 1558

Leisy, P. \& Dennefeld, M. 2006, A\&A, 456, 451

Magrini, L., Leisy, P., Corradi, R. L. M., et al. 2005, A\&A, 443, 115

Marigo, P. 2001, A\&A, 370, 194

Peña, M., Richer, M. G., \& Stasińska, G. 2007a, A\&A, 466, 75

Peña, M., Stasińska, G., \& Richer, M. G., 2007b, A\&A, 476, 745 